10 years ESJ

Special edition

\title{
Factores predictivos en el perfil de los jóvenes que interactúan en una situación de acoso en entornos escolares
}

\author{
Amaya Epelde-Larrañaga \\ Ligia Isabel Estrada-Vidal \\ University of Granada, Spain \\ Fátima Chacón-Borrego \\ University of Sevilla, Spain
}

Doi:10.19044/esj.2022.v18n5p117

Submitted: 30 November 2021

Accepted: 08 February 2022

Published: 21 February 2022
Copyright 2022 Author(s)

Under Creative Commons BY-NC-ND

4.0 OPEN ACCESS

Cite As:

Epelde-Larrañaga A., Estrada-Vidal L.I. \& Chacón-Borrego F. (2022). Factores predictivos en el perfil de los jóvenes que interactúan en una situación de acoso en entornos escolares. European Scientific Journal, ESJ, 18 (5), 117. https://doi.org/10.19044/esj.2022.v18n5p117

\section{Resumen}

Las situaciones de bullying y el cyberbullying destrozan la vida de los adolescentes provocando consecuencias nefastas. Es necesario tomar medidas para frenar estos actos de violencia. La educación de los adolescentes, los padres y los profesores es imprescindible para evitar estas situaciones. El objetivo del estudio: predecir qué factores influyen en los tres actores que intervienen en una situación de acoso (víctima, agresor y testigo), a partir del perfil de dichos actores en situación de espacios digitales, la edad y género del alumnado, su forma de trabajar en equipo y el tipo de centro escolar en el cual cursan sus estudios. Metodología: estudio no experimental de tipo cuantitativo en el que se realiza un análisis de regresión lineal múltiple. El estudio se ha llevado a cabo en dos centros educativos diferentes de la Ciudad de Melilla. La muestra ha estado compuesta por 227 escolares de entre 11 y 14 años. Los instrumentos utilizados han sido el cuestionario Cyberbullying: Screaning de acoso entre iguales (Garaigordóbil, 2013) y el Test de Trabajo en Equipo. Los principales resultados indican que el testigo en situación de acoso fue el predictor más fuerte para predecir la víctima en situación de acoso $(\beta=0,350)$, seguido de la víctima en situación de ciberacoso $(\beta=0,234)$ y el modelo predictivo del agresor en situación de acoso incluyó las variables predictivas agresor/a en situación de ciberacoso $(\beta=0,666)$ y testigo en situación de acoso 
$(\beta=0,144)$. Por tanto, se puede concluir que la conducta de los diferentes actores de bullying y cyberbullying está condicionada por la conducta de los demás actores, el agresor que agrede en una modalidad, agrede con facilidad en la otra y es más fácil agredir si existen testigos en estas situaciones de acoso o ciberacoso.

Palabras claves: Bullying, Cyberbullying, Educación, Predicción, Perfil de los participantes

\title{
Predictive Factors in the Profile of Young People who Interact in a Situation of Bullying in School Settings
}

\author{
Amaya Epelde-Larrañaga \\ Ligia Isabel Estrada-Vidal \\ University of Granada, Spain \\ Fátima Chacón-Borrego \\ University of Sevilla, Spain
}

\section{Abstract}

Bullying and cyberbullying situations destroy the lives of adolescents causing direct consequences. It is necessary to take measures to stop these acts of violence. The education of adolescents, parents and teachers is essential to avoid these situations. The objective of the study was to predict what factors influence the three participants involved in a bullying situation (victim, aggressor and witness), based on the profile of these participants in situations of digital spaces, age and gender of the students, their teamwork and the type of school in which they study. Methodology: non-experimental quantitative study in which a multiple linear regression analysis is performed. The study has been carried out in two different educational centers in the City of Melilla. The sample has been composed of 227 school children between 11 and 14 years old. The instruments used were the Cyberbullying questionnaire: Screening of harassment among equals (Garaigordóbil, 2013) and the Teamwork Test. The main results indicate that the witness in a bullying situation was the strongest predictor for predicting the victim in a bullying situation $(\beta=0.350)$, followed by the victim in a cyberbullying situation ( $\beta=$ 0.234 ) and the predictive model of the aggressor in bullying situation included the predictive variables aggressor in a cyberbullying situation $(\beta=0.666)$ and witness in a bullying situation ( $\beta=0.144)$. Therefore, it can be concluded that the behavior of the different participants of bullying and cyberbullying is conditioned by the behavior of the other participants, the aggressor who 
attacks in one modality, attacks easily in the other, and it is easier to attack if there are witnesses in these bullying or cyberbullying situations.

Keywords: Bullying, Cyberbullying, Education, Prediction, Profile of the participants

\section{Introduction}

El bullying y ciberbullying son la lacra social que invade y destroza la vida de muchos adolescentes (Carvalho, Branquinho and De Matos, 2017) y que se caracteriza por el abuso de poder de una persona o un grupo contra otra u otras personas con la finalidad de infligir daño (Modecki, Minchin, Harbaugh, Guerra, and Runions, 2014). Se visualiza a través de actuaciones agresivas que se repiten en el tiempo y que pueden ser de tipo físico, verbal, social y psicológico, como son la burla, la amenaza, la intimidación, la agresión física, los insultos... Cuando además todas estas actuaciones se llevan a cabo a través de las tecnologías cibernéticas, el daño que causan es todavía mayor que con el acoso tradicional, ya que esta vía tiene el poder de divulgar las agresiones por todo el mundo y durante el tiempo que el agresor quiera sin que la víctima pueda ejercer ningún control sobre la situación ni sobre su propia vida (Garaigordóbil, 2015; Navarro y Serna, 2016; Moore et al., 2017; Chacón-Cuberos, Castro-Sánchez, González-Campos y ZuritaOrtega, 2018).

Estos actos producen consecuencias nefastas en todos los agentes participantes en las agresiones, no solo en las víctimas. Tal como señala Delgado (2019, p.119) "quienes participan o padecen las consecuencias de este fenómeno se ven afectados en su tranquilidad, su autoestima, su emocionalidad y su proyección social, con posibles secuelas adversas para su integridad". Cuando pensamos en las consecuencias de estos actos, siempre, acudimos en primera instancia, a las relacionamos con las víctimas. Sin embargo, y aunque ellos no sean conscientes en el momento de la agresión, los agresores y los testigos sufren también en un futuro más bien cercano, las consecuencias de sus actos, teniendo en cuenta que los testigos son, de acuerdo con diferentes autores (Villanueva, Górriz y Adrián, 2008; Garaigordóbil, Mollo-Torrico y Larrain, 2019; Acevedo-Alemán y Cuellar-Fermín, 2020), agresores pasivos que no tomaron las medidas necesarias cuando observaban las agresiones. Los agresores activos y pasivos tienen un riesgo alto de sufrir consecuencias irrefrenables en poco tiempo teniendo en cuenta que además, todos estos agentes son adolescentes que se encuentran en una etapa vulnerable de su vida. Todos sufren antes o después, por tanto, se deben tomar medidas para frenar y erradicar estos actos.

Charlas de concienciación de los agentes participantes, intervenciones escolares para paliar situaciones violentas, análisis y prevención de actos 
violentos, son las medidas imprescindibles que se deben adoptar. Se debe comenzar en los centros educativos, debido a la edad de los agentes participantes ya que es en este entorno donde se reúnen, se conocen y se producen los ataques. La mayoría de los actos agresivos se llevan a cabo en los centros educativos, aunque después se extiende a otros lugares máxime cuando el ataque se realiza a través de medios cibernéticos.

La educación de los adolescentes es muy necesaria e importante y la formación de padres y profesores lo es también al mismo nivel. Según Ribeiro and Caliman (2015), Carvalho et al. (2017) y González-Torres, Vega-Campos y Mballa Louis (2017) el gobierno es el responsable de salvaguardar los intereses de los niños y debe garantizar sus derechos a través de la elaboración de leyes y documentos que lo avalen y prevengan la violencia entre iguales. Entre todos, se podrían evitar muchas situaciones violentas en las aulas si se tuviesen en su poder datos, observasen síntomas y sospecharan de la presencia de víctimas y agresores en sus aulas. También los padres y tutores legales deben saber reconocer los síntomas que caracterizan a las víctimas y agresores pasivos y activos, conversar con sus hijos, reflexionar con ellos...

Una técnica metodológica eficaz en las aulas para la detección de síntomas de acoso y la mitigación de conflictos es el trabajo en equipo, colaborativo o en grupo. Se trata de una estrategia utilizada por muchos profesores para facilitar el aprendizaje de algunos temas, y que precisa al mismo tiempo, de una interacción activa y positiva entre los adolescentes. Trabajar en grupo requiere habilidades sociales y emocionales que se deben adoptar para conseguir terminar con éxito una tarea y acorta la duración del trabajo si se ha llevado a cabo bajo una estrecha colaboración entre los miembros del grupo. Hoy en día vivimos en una era competitiva en la que la exigencia de un trabajo bien hecho y en el espacio de tiempo más corto posible es rigurosamente buscado (Dávila, 2016). El aprendizaje colaborativo potencia las cualidades sociales y la inteligencia emocional, lo que favorece las relaciones interpersonales creando un buen clima de aula y evitando los conflictos y las agresiones entre menores (León, Gozalo y Polo, 2012; Chacón-Cuberos et al., 2018). Por tanto, el trabajo en grupo de manera colaborativa es un recurso para reducir o evitar las situaciones violentas (Castro Morales, 2011; Van Ryzin y Roseth, 2018).

Como antecedentes del presente estudio, se ha llevado a cabo en dos centros educativos de la Ciudad Autónoma de Melilla una investigación sobre el bullying y el Ciberbullying entre adolescentes con el fin de disminuir los casos y mejorar la convivencia entre ellos. Los resultados (Chacón-Cuberos, Epelde-Larrañaga y Oñederra-Ramírez, 2021; Epelde-Larrañaga, ChacónCuberos y Oñederra-Ramírez, 2019a; Epelde-Larrañaga, Oñederra-Ramírez y Chacón-Cuberos, 2019b; Epelde-Larrañaga, Oñederra-Ramírez y ChacónCuberos, 2020a; Epelde-Larrañaga, Oñederra-Ramírez y Estrada-Vidal, 
2020b) fueron encaminados a determinar la incidencia del bullying, del ciberbullying, y de las habilidades de los estudiantes en la técnica del trabajo en equipo durante el pretest y revelaron que la incidencia del bullying y cyberbullying era muy acusada en los dos centros educativos investigados y los estudiantes declaraban sobre todo, ser testigos de estas agresiones.

Posteriormente, se realizó una intervención de carácter musical y una charla sobre valores morales en cada centro y se analizó de nuevo la incidencia de los tres mismos aspectos para ver los cambios que se habían producido. El postest proporcionó una disminución de los niveles de bullying y ciberbullying en los dos centros. En el concertado, se produjo una gran disminución de situaciones de ciberbullying y también de acoso presencial aunque en menor medida. En el centro público, la victimización en acoso presencial disminuyó considerablemente (Epelde-Larrañaga et al., 2020b).

Ante esta mejoría en el perfil de los tres actores de una situación de acoso y ciberacoso, se consideró relevante conocer qué factores podían influir o predecir el perfil del alumnado en una situación de acoso (como víctima, como agresor y como testigo) a partir de la interactuación que pueden tener de manera tanto presencial como en las redes sociales. Así, los posibles factores a tener en cuenta son el perfil de cada uno de los tres actores en situación presencial como cibernética.

A ellos, se suma el género y edad del alumnado, puesto que en otros estudios se han comprobado diferencias (Benítez and Justicia, 2006; Cerezo, 2009; Garaigordóbil, 2015; Sáenz and Vergara, 2016; Menesini and Salmivalli, 2017; Triviño García, Iriarte Corpas y González Gris, 2017; Mizuta, Okada, Nakamura, Yamaguchi y Ojima, 2018; Athanasiou et al., 2018; Yudes Gómez, Baridon Chauvie y González Cabrera, 2018), y debido a que se tienen distintos idearios según el tipo de centro, también se consideró como factor. Finalmente, el tipo de perfil del alumnado dentro del trabajo en equipo, también puede ser un condicionante, en cuanto que se pueden generar ciertas conductas favorables hacia las relaciones sociales, y por tanto, su predisposición a ser un actor dentro de las situaciones de acoso.

Es por ello, que se plantea como objetivo de estudio predecir qué factores influyen en los tres actores que intervienen en una situación de acoso (víctima, agresor y testigo), a partir del perfil de dichos actores en situación de espacios digitales, la edad y género del alumnado, su forma de trabajar en equipo y el tipo de centro escolar en el cual cursan sus estudios.

\section{Método}

Diseño de investigación de corte no experimental, transversal, cuantitativo tipo encuesta en el que se realizó un análisis de regresión lineal múltiple. 


\section{Participantes}

El estudio se ha realizado en dos centros educativos de la ciudad de Melilla (La Salle-El Carmen y el Instituto público de Educación Secundaria Enrique Nieto) cuyo contexto alberga una gran diversidad cultural debido a la población que convive, pero también a la afluencia de personas migrantes que residen temporalmente en la ciudad hasta el momento en el cual pueden ir a residir a otras localidades europeas. Así, en los centros escolares de Melilla se encuentran representados todos ellos, como derecho constitucional que tiene todo ciudadano a la educación. Aunque la Administración local permite investigar en los 23 centros educativos de la ciudad, no dan autorización para recoger información sobre el tipo de religión (cristiana, musulmana, hebrea, hindú...), origen étnico (europeo, bereber, israelita...), la lengua materna (español, tamazight, hebreo,...).

La muestra seleccionada fue de 227 estudiantes de los cursos de $6^{\circ}$ de Educación Primaria y $1^{\mathrm{o}}$ de Educación Secundaria. El error muestral fue de 0,$05 ; \mathrm{IC}=90 \% ; 54,6 \%$ alumnas y $45,4 \%$ alumnos, con una media de edad de 12,06 años (DT=0,771).

\section{Instrumentos}

Para la recogida de información se utilizaron el Test Cyberbullying de Garaigordóbil (2013) en su versión al español, el test del Trabajo en Equipo (Epelde-Larrañaga et al., 2019a) de elaboración propia y validado por un panel de expertos y la Hoja de Auto-Registro para evaluar variables sociodemográficas.

El primer instrumento presenta dos secciones. La primera tiene 12 items para recoger información sobre el acoso, en el cual se distinguen cuatro tipos de acoso presencial (físico, verbal, social y psicológico) agrupados según el rol de la situación de acoso (víctima, agresor y observador), respondiéndose desde los tres roles de forma triangular. La segunda tiene 45 ítems para recoger información sobre el acoso en entornos virtuales (cyberbullying), en el cual se evalúan 15 tipos de conducta (cinco en cada uno de los tres roles mencionados). La escala es de tipo Likert con cuatro opciones $(0=$ nada a $3=$ siempre), cuya sumatoria de las puntuaciones obtenidas en cada una de las secciones permite la clasificación de los diversos roles. El coeficiente alfa de Cronbach en la sección de acoso es de 0,81 y de 0,91 para la de ciberbullying, considerándose así, buena la consistencia interna del instrumento (Garaigordóbil, 2013).

Test de Trabajo en Equipo. Se utiliza un cuestionario de elaboración propia, el cual fue validado mediante panel de expertos. Este cuestionario se compone de 14 ítems que puntúan diferentes aspectos de la capacidad de los encuestados para trabajar en grupo (p.e. "Contribuyes a que la comunicación entre los miembros del grupo sea clara y fluida). Estos son puntuados mediante 
una escala tipo Likert de cuatro opciones donde $1=$ Nunca y $4=$ Siempre. Para este instrumento se obtuvo una consistencia interna de $\alpha=0,806$, la cual es aceptable.

Hoja de auto-registro. Se emplea un cuestionario tipo Ad Hoc para el registro de variables de tipo sociodemográfico, tales como el sexo, la edad, el centro educativo o el curso.

\section{Procedimiento}

La recogida de información dio comienzo con la solicitud de participación a los equipos directivos de los centros, a partir de los cuales, se les hacía llegar la solicitud a los docentes de las clases de interés (técnica de muestreo no probabilístico intencional). Al equipo directivo se le facilitó la información pertinente sobre el estudio. Se solicitó el consentimiento firmado de las familias de los participantes en el estudio para poder recoger la información e intervenir sobre los mismos, siempre en horario lectivo y con la presencia de la investigadora principal. Se siguieron los principios generales de ética científica, respetando a los participantes, su autonomía para participar (voluntaria), su anonimato, o la no maleficencia, entre otros (Declaración de Helsinki).

\section{Análisis estadístico}

Se ha realizado un análisis de regresión lineal múltiple con el método stepwise, considerando como variable criterio el perfil de la víctima, del agresor y de los testigos en una situación de acoso, respectivamente en cada uno de los análisis. Como variables predictores se hizo uso de los tres perfiles en situaciones de acoso y de ciberacoso (se omitía como variable predictor la que actuaba como variable dependiente en el análisis), el trabajo en equipo, el género y edad de los participantes, así como el tipo de centro (privado o público) (Figura 1). Los análisis permiten directamente conocer los supuestos del modelo excepto el de homocedasticidad, por lo que se comprobó con la prueba de Leven para conocer si $\mathrm{p}>0,05$.

Los datos fueron analizados por medio del software estadístico IBM SPSS $® 25.0$ 
Figura 1 - Supuesto de normalidad: Histograma y Gráfico de probabilidad normal de las variables

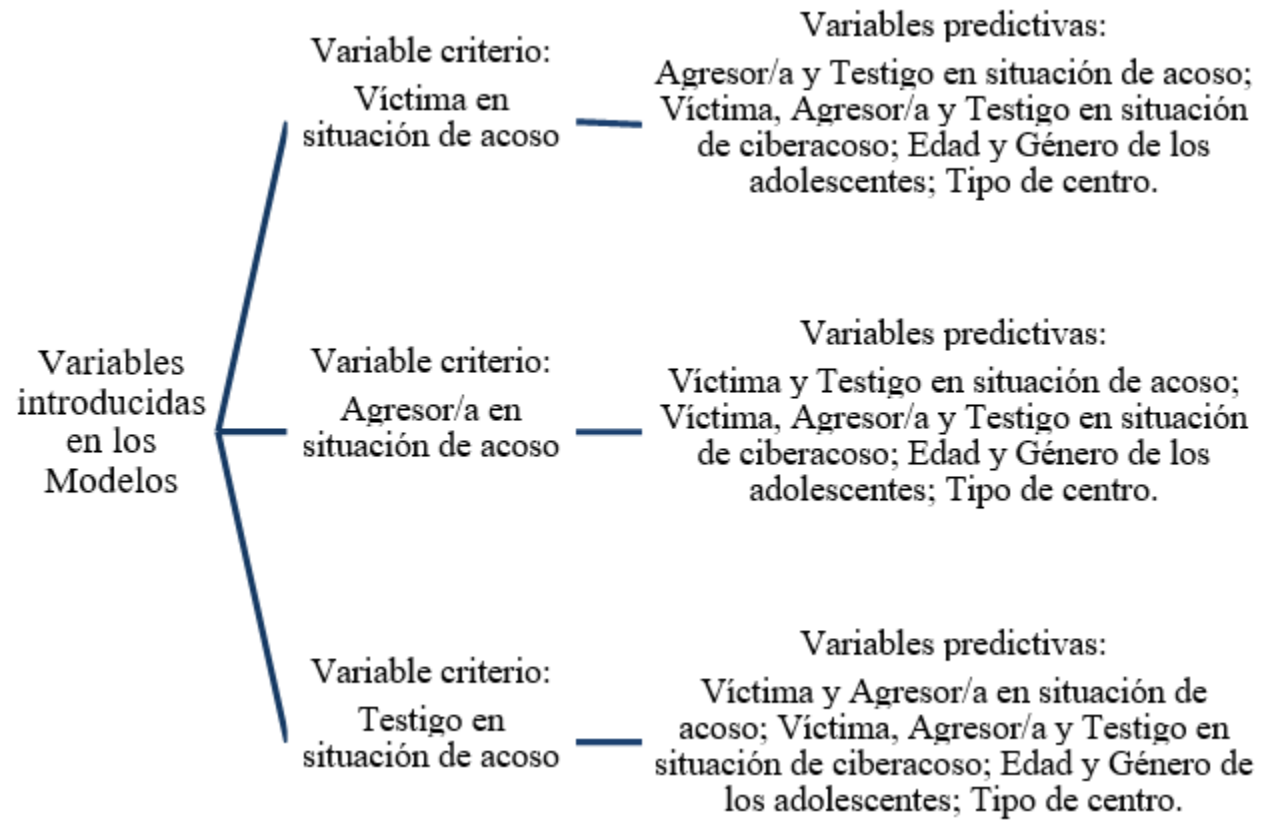

\section{Resultados}

Los resultados a partir del análisis de regresión lineal múltiple, indicaron que se cumplían los supuestos del modelo, y por tanto, la validez del modelo que predice el perfil de la víctima, del o la acosador/a y del testigo en situación de acoso (Aragón et al., 2015). Así, se verificó que los tres modelos cumplían con el supuesto del modelo (Vilá et al., 2019). En concreto, el supuesto de linealidad, de independencia de los errores (Durbin-Watson está entre 1,5 y 2,5; Tablas 1, 3 y 5), de normalidad (Figura 2), de homocedasticidad ( $p>0,05$ ), de no colinealidad (tolerancia mayor a 0,10 , y el factor de inflación de la varianza inferior a 10; Tablas 2, 4 y 6). 
European Scientific Journal, ESJ ISSN: 1857-7881 (Print) e - ISSN 1857-7431 February 2022

Bridging Language, Medicine, and Law

Figura 2 - Supuesto de normalidad: Histograma y Gráfico de probabilidad normal de las variables criterio víctima, agresor y testigo en situación de acoso

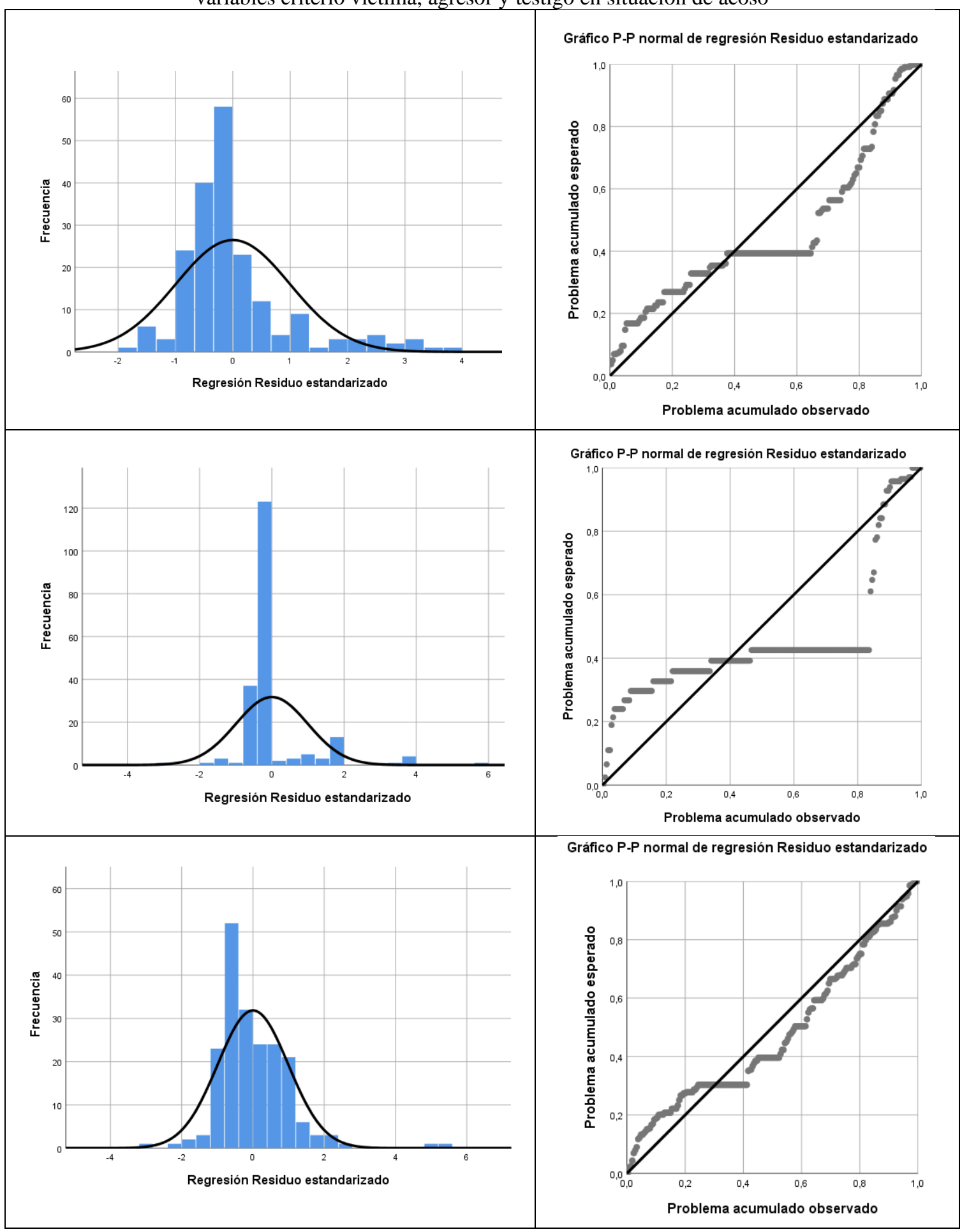


El análisis de regresión lineal múltiple sugirió 2 modelos en el caso de las variables criterio víctima y agresor en situación de acoso, y 3 modelos en el caso de la variable criterio testigo en situación de acoso. Respectivamente, los modelos que mayor capacidad explicativa ofrecieron fueron el modelo 2, el modelo 2 y el modelo 3 (Tabla 1, 2 y 3). La bondad de ajuste del modelo que corresponde a las variables criterio víctima y testigo, fueron poco adecuados (respectivamente, las variables introducidas en el modelo explicaron el $22,5 \%$ y $31,8 \%$ de la varianza de las variables criterio). En el caso de la variable criterio del/la agresor/a en situación de acoso, este fue explicado por las variables predictivas en un $48,1 \%$ de la varianza.

Tabla 1- Modelo de Regresión Lineal Múltiple por pasos para predecir el perfil de la víctima en situaciones de acoso

\begin{tabular}{cccccrrrr}
\hline Modelo $^{\mathrm{c}}$ & $R$ & $R^{2}$ & $\begin{array}{c}R^{2} \\
\text { corregida }\end{array}$ & $\begin{array}{c}\text { Error típ } \\
\text { estimación }\end{array}$ & g.l. & $F$ & $p$ de $F$ & $\begin{array}{c}\text { Dubin- } \\
\text { Watson }\end{array}$ \\
\hline 1 & $0,430^{\mathrm{a}}$ & 0,185 & 0,181 & 1,1678 & 1 & 44,430 & 0,001 & \\
\hline 2 & $0,483^{\mathrm{b}}$ & 0,233 & $\mathbf{0 , 2 2 5}$ & 1,1357 & 2 & 29,616 & 0,001 & 1,625 \\
\hline
\end{tabular}

Nota - (a) Variables predictoras: (Constante), Testigos en situación de acoso; (b) Variables predictoras: (Constante), Testigos en situación de acoso, Víctima en situación de ciberacoso;

(c) Variable dependiente: Víctima en situación de acoso.

Tabla 2 - Modelo de Regresión Lineal Múltiple por pasos para predecir el perfil de la víctima en situaciones de acoso

\begin{tabular}{ccccccccc}
\hline Modelo $^{\mathrm{c}}$ & $R$ & $R^{2}$ & $\begin{array}{c}R^{2} \\
\text { corregida }\end{array}$ & $\begin{array}{c}\text { Error típ } \\
\text { estimación }\end{array}$ & g.l. & $F$ & $p$ de $F$ & $\begin{array}{c}\text { Dubin- } \\
\text { Watson }\end{array}$ \\
\hline 1 & $0,683^{\mathrm{a}}$ & 0,466 & 0,463 & 0,4892 & 1 & 171,039 & 0,001 & \\
\hline 2 & $0,697^{\mathrm{b}}$ & 0,486 & $\mathbf{0 , 4 8 1}$ & 0,4810 & 2 & 92,325 & 0,001 & 1,888 \\
\hline
\end{tabular}

Nota. (a) Variables predictoras: (Constante), Agresor en situación de ciberacoso; (b) Variables predictoras: (Constante), Agresor en situación de ciberacoso, Testigos en situación de acoso; (c) Variable dependiente: Agreso en situación de acoso.

Tabla 3 - Modelo de Regresión Lineal Múltiple por pasos para predecir el perfil del testigo en situaciones de acoso

\begin{tabular}{cccccrrrr}
\hline Modelo $^{\mathrm{d}}$ & $R$ & $R^{2}$ & $\begin{array}{c}R^{2} \\
\text { corregida }\end{array}$ & $\begin{array}{c}\text { Error típ } \\
\text { estimación }\end{array}$ & g.l. & $F$ & $p$ de $F$ & $\begin{array}{c}\text { Dubin- } \\
\text { Watson }\end{array}$ \\
\hline 1 & $0,459^{\mathrm{a}}$ & 0,211 & 0,207 & 2,0533 & 1 & 52,446 & 0,001 & \\
\hline 2 & $0,553^{\mathrm{b}}$ & 0,306 & 0,299 & 1,9312 & 2 & 42,921 & 0,001 & \\
\hline 3 & $0,573^{\mathrm{c}}$ & 0,328 & $\mathbf{0 , 3 1 8}$ & 1,9045 & 3 & 31,595 & 0,001 & 1,963 \\
\hline
\end{tabular}

Nota - (a) Variables predictoras: (Constante), Testigos en situación de ciberacoso; (b) Variables predictoras: (Constante), Testigos en situación de ciberacoso, Víctima en situación de acoso; (b) Variables predictoras: (Constante), Testigos en situación de ciberacoso, Víctima en situación de acoso, Edad de los participantes; (d) Variable dependiente: Testigo en situación de acoso.

En el modelo predictivo de la víctima en situación de acoso, se encontraron dos variables introducidas en el modelo, y que fueron, el perfil del testigo en situación de acoso y de la víctima en situación de ciberacoso. Así, quedaron excluidas las demás variables. Dicho modelo predictivo 
presentó un valor de $t$ que se asocia a una probabilidad de error inferior a 0,05 en las dos variables incluidas en el modelo (Tabla 2).

En otro sentido, los coeficientes estandarizados indican qué variables presentan un mayor peso explicativo en el modelo. En concreto, el testigo en situación de acoso fue el predictor más fuerte para predecir la víctima en situación de acoso $(\beta=0,350)$, seguido de la víctima en situación de ciberacoso $(\beta=0,234)$ (Tabla 4). Por tanto, se puede decir que cuanto más actúe otro/s adolescente/s con el rol de testigo en una situación de acoso presencial y cuanto más esté sufriendo acoso en un entorno virtual, más ejercerá el rol de víctima en situaciones presenciales.

Tabla 4 - Coeficientes en la ecuación del Modelo de Regresión Lineal Múltiple para predecir el rol de la víctima en situaciones de acoso

\begin{tabular}{|c|c|c|c|c|c|c|c|c|}
\hline & \multirow{2}{*}{ Modelo } & \multicolumn{2}{|c|}{$\begin{array}{l}\text { Coeficientes no } \\
\text { estandarizados }\end{array}$} & \multirow{2}{*}{$\begin{array}{c}\begin{array}{c}\text { Coeficientes } \\
\text { estandarizados }\end{array} \\
\beta\end{array}$} & \multirow{2}{*}{$t$} & \multirow{2}{*}{$P$} & \multicolumn{2}{|c|}{$\begin{array}{l}\text { Estadísticas de } \\
\text { colinealidad }\end{array}$} \\
\hline & & B & $\begin{array}{l}\text { Desv. } \\
\text { Error }\end{array}$ & & & & Tolerancia & VIF \\
\hline \multirow[t]{3}{*}{$2^{a}$} & (Constante) & 0,309 & 0,104 & & 2,962 & 0,003 & & \\
\hline & $\begin{array}{l}\text { Testigo } \\
\text { Acoso }\end{array}$ & 0,196 & 0,037 & $\mathbf{0 , 3 5 0}$ & 5,243 & 0,001 & 0,883 & 1,133 \\
\hline & $\begin{array}{c}\text { Víctima } \\
\text { Ciberacoso }\end{array}$ & 0,118 & 0,034 & 0,234 & 3,500 & 0,001 & 0,883 & 1,133 \\
\hline
\end{tabular}

(a) Variable dependiente: Perfil de la víctima en situación de acoso.

El modelo predictivo del agresor en situación de acoso incluyó las variables predictivas agresor/a en situación de ciberacoso $(\beta=0,666)$ y testigo en situación de acoso $(\beta=0,144)$, cuyo valor de $t$ se asoció a una probabilidad de error inferior a 0,05 (Tabla 5).

Tabla 5 - Coeficientes en la ecuación del Modelo de Regresión Lineal Múltiple para predecir el perfil del agresor en situaciones de acoso

\begin{tabular}{|c|c|c|c|c|c|c|c|c|}
\hline & \multirow{2}{*}{ Modelo } & \multicolumn{2}{|c|}{$\begin{array}{l}\text { Coeficientes no } \\
\text { estandarizados }\end{array}$} & \multirow{2}{*}{$\begin{array}{c}\begin{array}{c}\text { Coeficientes } \\
\text { estandarizados }\end{array} \\
\beta\end{array}$} & \multirow{2}{*}{$t$} & \multirow{2}{*}{$p$} & \multicolumn{2}{|c|}{$\begin{array}{c}\text { Estadísticas de } \\
\text { colinealidad }\end{array}$} \\
\hline & & B & $\begin{array}{l}\text { Desv. } \\
\text { Error }\end{array}$ & & & & Tolerancia & VIF \\
\hline $2^{a}$ & (Constante) & 0,091 & 0,044 & & 2,063 & 0,040 & & \\
\hline & $\begin{array}{c}\text { Agresor } \\
\text { Ciberacoso }\end{array}$ & 0,346 & 0,027 & 0,666 & 12,886 & 0,001 & 0,986 & 1,014 \\
\hline & $\begin{array}{l}\text { Testigo } \\
\text { Acoso } \\
\end{array}$ & 0,042 & 0,015 & 0,144 & 2,781 & 0,006 & 0,986 & 1,014 \\
\hline
\end{tabular}

(a) Variable dependiente: Perfil del agresor en situación de acoso.

Finalmente, en la Tabla 6 se puede consultar también un valor de $t$ asociado a una probabilidad de error inferior a 0,05 en el modelo predictivo del testigo en situación de acoso. En este caso, el predictor que presentó más fuerza fue el testigo en situación de ciberacoso $(\beta=0,361)$, seguido de la víctima de acoso $(\beta=0,319)$ y la edad de los adolescentes $(\beta=0,150)$. Es decir, 
que el alumno/a actuará en situaciones de acoso presencial cuanto más acostumbrado esté de hacerlo en situaciones virtuales, y cuanto más ejerza la víctima su papel en dicha situación. Además, aunque influirá en mucha menor intensidad, también se potenciará su papel de testigo cuanta más edad tenga.

Tabla 6 - Coeficientes en la ecuación del Modelo de Regresión Lineal Múltiple para predecir el perfil del testigo en situaciones de acoso

\section{Coeficientes no Coeficientes}

estandarizados estandarizados
B

Desv. $\beta$

\section{Estadísticas de} colinealidad

Tolerancia VIF

\begin{tabular}{|c|c|c|c|c|c|c|c|c|}
\hline $3^{\mathrm{a}}$ & (Constante) & $-4,771$ & 2,254 & & $-2,117$ & 0,036 & & \\
\hline & $\begin{array}{c}\text { Testigo } \\
\text { Ciberacoso }\end{array}$ & 0,205 & 0,035 & 0,361 & 5,865 & 0,001 & 0,912 & 1,097 \\
\hline & $\begin{array}{l}\text { Víctima } \\
\text { Acoso }\end{array}$ & 0,570 & 0,110 & 0,319 & 5,179 & 0,001 & 0,912 & 1,097 \\
\hline & Edad & 0,479 & 0,188 & 0,150 & 2,553 & 0,011 & 0,999 & 1,001 \\
\hline
\end{tabular}

(a) Variable dependiente: Perfil del testigo en situación de acoso.

Según lo expuesto, en el caso de los modelos que predicen el comportamiento de la víctima y el testigo en situaciones presenciales de acoso, son muy adecuados. Sin embargo, el mejor modelo fue el que predijo el comportamiento del agresor en situación de acoso. En este caso, se puede decir que actuará como agresor/a en el entorno presencial con otros adolescentes cuanto más habituado esté de desempeñar dicho rol en el entorno virtual, y aunque la relación es mucho menor, también, cuanto más actúa como víctima el adolescente sobre el cual está realizando el acto de acoso.

\section{Discusión}

El diseño e implementación del presente proyecto de investigación mediante un diseño cuasiexperimental, ha permitido abordar la problemática del acoso y ciberacoso en centros educativos de la ciudad de Melilla. Inicialmente, se ha podido identificar el grado del rol que presenta el alumnado objeto de estudio en acoso y ciberacoso (víctima, agresor/a y testigo) (ChacónCuberos et al., 2021; Epelde-Larrañaga et al., 2019a; Epelde-Larrañaga et al., 2019b; Epelde-Larrañaga et al., 2020a). Posteriormente, se ha podido comprobar que hay una mejora en la actitud y comportamiento del alumnado cuando se intervienen educativamente en el mismo mediante el trabajo en grupo que se realiza al ensayar grupalmente para interpretar una obra musical, como integrantes de un grupo musical dentro de la asignatura de educación musical, y complementado con la asistencia a un taller para concienciar sobre la problemática de acoso y ciberacoso (Epelde-Larrañaga et al., 2020b).

Sin embargo, las inquietudes que surgieron del proyecto se ampliaron con el fin de conocer si hay variables relacionadas con los perfiles del alumnado en situaciones de acoso, y qué factores en concreto pueden predecir 
su comportamiento. Hay varias investigaciones que han buscado variables predictores del ciberbullying. Felipe-Castaño, León del Barco y Fajardo (2013) aseguran que los alumnos que intervienen en casos de acoso sufren, a lo mínimo, un malestar psicológico. En esta línea muchos autores hablan de la desconexión moral, la insensibilidad, los bajos niveles de inteligencia emocional, falta de valores morales, emociones morales y remordimiento que presentan los adolescentes (Garaigordóbil y Oñederra, 2010; Perren and Gutzwiller Helfenfinger, 2012; Inglés et al., 2014; Orue and Calvete, 2019; Flores, Caballer y Romero, 2019; Wang and Ngai, 2020). Así mismo, otros autores, (Prodócimo, Cerezo y Arense, 2014; Garaigordóbil y Machimbarrena, 2019) van más adelante confrontando algunas de las posibles causas de este malestar achacándolo a los problemas sociofamiliares; afirman que las familias desestructuradas, el número elevado de hermanos, el bajo nivel de formación de los padres, los padres autoritarios, padres que no apoyan a sus hijos y otras acciones de este tipo, pueden provocar las situaciones de acoso.

Nuestro interés recae en estudiar si la conducta de los distintos actores está condicionada por la conducta del resto de actores en situaciones de acoso y ciberacoso. Además, a ello se suma que la edad y género del alumnado puede determinar en cierta medida dichas conductas, tal como muchos autores atestiguan (Caballo, Arias, Calderero, Salazar y Irurtia, 2011; Beckman, Hagquist and Hellström, 2013; Chocarro y Garaigordóbil, 2019), así como también el tipo de centro por tener estos un ideario educativo que los caracteriza (Carreres y Sánchez, 2016; Arufe-Giráldez, Chacón-Cuberos, Zurita-Ortega, Lara-Sánchez and Castro-García, 2017).

Es por ello, que desde el proyecto de investigación también se ha podido conocer que la conducta del agresor en situaciones presenciales de acoso se ve reforzada por su conducta en las redes virtuales. Es decir, cuanto más habituado está a desempeñar su rol de agresor/a en el entorno virtual, más fácil será que lo desempeñe en situaciones presenciales al reforzar una conducta de intimidación sobre los iguales. Este resultado coincide con estudios de varios autores (Orue and Calvete, 2019; Perren and Gutzwiller Helfenfinger, 2012). También encontramos, aunque su relación está en mucha menor medida, que el agresor ejerce su rol con más facilidad, en caso de actuar otros iguales como testigos durante situaciones de acoso. No existen estudios que trabajen el rol de testigos en los casos de bullying y cyberbullying, pero se aconseja que en los programas de sensibilización se les incluya.

Las futuras líneas de trabajo se conducirán hacia la necesidad de realizar intervenciones en los centros para disminuir los conflictos, en los que se potencia el trabajo colaborativo y la utilización de recursos, como son las actividades musicales y los juegos (Chacón-Borrego, Corral-Pernía y Del Rey, 2020), además de la implementación de programas específicos de intervención y prevención como los propuestos por Ortega-Ruiz, Rey and Casas (2012) o 
Garaigordóbil y Martínez-Velderrey (2016), para influir en las emociones de las personas e intentar reducir los niveles de agresión a partir del desarrollo de la capacidad de empatía. También creemos necesario seguir ampliando las investigaciones en este ámbito, que permitan realizar predicciones de las actitudes de acoso entre iguales y de esta forma poder diseñar programas de prevención más eficaces.

\section{Conclusiones}

De acuerdo con el objetivo planteado en el estudio, existen variables relacionadas con los perfiles de los actores del acoso y ciberacoso, como la edad, el sexo, el tipo de centro. La conducta de los diferentes actores de bullying y cyberbullying está condicionada por la conducta de los demás actores. El agresor que agrede en una modalidad, agrede con facilidad en la otra. El agresor ejerce su rol con más facilidad si existen testigos en esta situación de acoso o ciberacoso.

Los centros educativos tienen un papel fundamental en la educación y concienciación de los estudiantes para evitar situaciones violentas. Es necesario intervenir educativamente para mejorar sus conocimientos, valores, actitudes, y hábitos, con el fin de mejorar sus conductas hacia una convivencia más pacífica de calidad para todos. En la oferta educativa de los centros educativos debe primar la humanidad, la educación emocional, el aprendizaje cooperativo y la educación en valores. Consideramos que es necesario reforzar desde edades tempranas todos estos aspectos y continuar trabajándolos en etapas educativas superiores. Esta educación junto al afecto parental y su involucración en la vida de los menores, el diálogo y la comunicación son factores importantes para disminuir el problema. Para ello, es importante, la formación continua del profesorado, especialmente de los tutores/as, y de los padres del alumnado, como factor fundamental de prevención y paliación de situaciones de acoso y ciberacoso.

\section{Agradecimiento}

Es una investigación aprobada por la Dirección Provincial de Educación de Melilla titulada: "Estudio Piloto para evaluar y mejorar la convivencia entre iguales en Centros de Primaria y Secundaria de Melilla por medio de la Música". Agradecemos su colaboración a todos los participantes en esta investigación, y sobre todo, a los profesores de los centros educativos estudiados.

\section{References:}

1. Acevedo-Alemán, J. y Cuellar-Fermín, K.C. (2020). El lado oculto del bullying: Los espectadores. Retos del trabajo social. Revista 
Internacional de trabajo social y ciencias sociales, 19, 9-2 http://revistas.uned.es/index.php/comunitania/article/view/26636.

2. Aragón, E.L., Navarro, J.I., Aguilar, M., and Cerda, G. (2015). Predictores cognitivos del conocimiento numérico temprano en alumnado de 5 años. Rev. Psicodidáctica, 20(1), 83-97. doi: 10.1387/RevPsicodidact.11088.

3. Arufe-Giráldez, V., Chacón-Cuberos, R., Zurita-Ortega, F., LaraSánchez, A., and Castro-García, D. (2017). Influence of Type of School on Sport Practice and Leisure Activities of School children. Educare Electronic Journal, 21(1), 1-19. doi: 10.15359/ree.21-1.6.

4. Athanasiou, K., Melegkovits, E., Andrie, E.K., Magoulas, C., Tzavara, C.K., Richardson, C.... Tsitsika, A.K. (2018). Cross-national aspects of cyberbullying victimization among 14-17-year-old adolescents across seven European countries. BMC Public Health. 18. doi:10.1186/s12889-018-5682-4.

5. Beckman, L., Hagquist, C., and Hellström, L. (2013). Discrepant gender patterns for cyberbullying and traditional bullying-An analysis of Swedish adolescent data. Computers in Human Behavior, 29 (5), 1896-1903. doi: 10.1016/j.chb.2013.03.010.

6. Benítez, J. L. y Justicia, F. (2006). El maltrato entre iguales: descripción y análisis del fenómeno. Electronic Journal of Research in Educational Psychology, 4(2), 151-170. https://www.researchgate.net/publication/28127157_El_maltrato_entr e_iguales_descripcion_y_analisis_del_fenomeno.

7. Caballo, V.E., Arias, B., Calderero, M., Salazar, I.C. y Irurtia, M.J. (2011). Acoso escolar y ansiedad social en niños (I): Análisis de su relación y desarrollo de nuevos instrumentos de evaluación. Psicología Conductual, 19(3), 591-609. https://www.behavioralpsycho.com/wpcontent/uploads/2019/08/06.Caballo_1_19-3oa.pdf.

8. Carreres, A. y Sánchez, P. (2016). Evaluación de las prácticas educativas del profesorado de los centros escolares: indicadores de mejora desde la educación inclusiva. REICE. Revista Iberoamericana sobre Calidad, Eficacia y Cambio en Educación, 8(5), 97-109. http://www.redalyc.org/articulo.oa?id=55119084007.

9. Carvalho, M.; Branquinho, C. and De Matos, M.G. (2017). Bullies, Victims and Provocative Victims in Context: Discriminant Factors in a Portuguese Adolescent Sample. European Scientific Journal, ESJ, 13(20). doi: 10.19044/esj.2017.v13n20p23.

10. Castro-Morales, J. (2011). Acoso escolar. Revista de Neuropsiquiatría, 74(2), 242-249. 
11. Cerezo, F. (2009). Bullying: análisis de la situación en las aulas españolas. International Journal of Psychology and Psychological Therapy, 9(3), 383-394. doi: 10.20453/mp.v74i2.1681.

12. Chacón-Borrego, F., Corral-Pernía, J.A. y Del Rey, R. (2020). Beneficios de la actividad física en la prevención del acoso escolar y la exclusión. En R. Ortega-Ruiz y F. Córdoba-Alcaide (Ed.). Educación Física y convivencia: oportunidades y desafíos en la prevención del acoso escolar (pp. 51-72). Editorial La Muralla.

13. Chacón-Cuberos, R., Castro-Sánchez, M., González-Campos, G. y Zurita-Ortega, F. (2018). Victimización en la escuela, ocio digital e irritabilidad: análisis mediante ecuaciones estructurales. RELIEVE, 24(1), 1-12

https://dialnet.unirioja.es/servlet/articulo?codigo $=6485215$.

14. Chacón-Cuberos, R., Epelde-Larrañaga, A. y Oñederra-Ramírez, J.A. (2021). Trabajo colaborativo en el aula, bullying y cyberbullying: Estudio con Adolescentes. Revista Iberoamericana de Psicología 12(3), 3-8.

https://reviberopsicologia.ibero.edu.co/article/view/rip.12301

15. Chocarro, E. y Garaigordóbil, M. (2019). Bullying y cyberbullying: diferencias de sexo en víctimas, agresores y observadores. Pensamiento Psicológico, 17(2), 57-71. doi: 10.11144/Javerianacali.PPSI17-2.bcds

16. Dávila, E. (2016). Rápido a ninguna parte. Consideraciones en torno a la aceleración del tiempo social. Acta Sociológica, 69, 51-75. http://www.revistas.unam.mx/index.php/ras/article/view/54280

17. Delgado Santamaría, O.Y. (2019). El lenguaje de las emociones: condiciones para la ciberciudadanía «más allá del cyberbullying».https://repositorio.idep.edu.co/bitstream/handle/001/1 904/Sistematizacion_Acompanamiento_In_Situ_p_203-

210.pdf?sequence $=1 \&$ is Allowed $=\mathrm{y}$

18. Epelde-Larrañaga, A., Chacón-Cuberos, R. y Oñederra-Ramírez, J.A. (2019a). El trabajo colaborativo en Primaria y Secundaria: Diferencias según sexo y curso. Acta Sociológica, 79, 11-32. doi: 10.22201/fcpys.24484938e.2019.79.72524

19. Epelde-Larrañaga, A., Oñederra-Ramírez, J.A. and Chacón-Cuberos, R. (2019b). Prevalence of Cyberbullying Data from Two Education Centres in Spain. The International Journal of Humanities Education, 17(1): 74-85.

https://www.researchgate.net/publication/348686139_Prevalence_of_ Cyberbullying_Data_from_two_Education_Centres_in_Spain

20. Epelde-Larrañaga, A., Oñederra-Ramírez, J.A., and Chacón-Cuberos, R. (2020a). The prevalence of bullying in two educational centers in 
Melilla (Spain). PSOCIAL, 6(2). https://publicaciones.sociales.uba.ar/index.php/psicologiasocial/articl e/view/4992

21. Epelde-Larrañaga, A.; Oñederra-Ramírez, J.A. and Estrada-Vidal, L.I. (2020b). Music as a Resource Against Bullying and Cyberbullying: Intervention in two Centers in Spain. Sustainability, 12, 2057. doi: $10.3390 / \mathrm{su} 12052057$

22. Felipe-Castaño, E.; León del Barco, B y Fajardo, F. (2013). Perfiles psicopatológicos de los participantes en situaciones de acoso escolar en Educación Secundaria. Psicología conductual, 21(3), 475-490. https://www.behavioralpsycho.com/producto/perfiles-

psicopatologicos-de-los-participantes-en-situaciones-de-acosoescolar-en-educacion-secundaria/

23. Flores, R., Caballer, A., y Romero, M. (2019). Efecto de un programa de prevención de ciberacoso integrado en el currículum escolar de Educación Primaria. Rev. Psicodidáctica, 25(1). doi: 10.1016/j.psicod.2019.08.001

24. Garaigordóbil, M. (2013). Cyberbullying: Screening de acoso entre iguales. TEA.

25. Garaigordobil, M. y Oñederra, J. A. (2010). La violencia entre iguales: Revisión teórica y estrategias de intervención. Pirámide.

26. Garaigordóbil, M. (2015). Cyberbullying en adolescentes y jóvenes del País Vasco: Cambios con la edad. Anales de Psicología, 31(3), 10691076. doi:10.6018/analesps.31.3.179151

27. Garaigordobil, M., and Martínez-Valderrey, V. (2016). Impact of Cyberprogram 2.0 on different types of school violence and aggressiveness. Frontiers in Psychology, 7, 428. doi: 10.3389/fpsyg. 2016.0042

28. Garaigordóbil, M., Mollo-Torrico, J.P. y Larrain, E. (2019).Prevalencia de Bullying y Cyberbullying en Latinoamérica: una revisión. Revista Iberoamericana de Psicología: Ciencia y Tecnología, 11(3), 1-18. doi: 10.33881/2027-1786.rip.11301

29. Garaigordóbil, M. and Machimbarrena, J.M. (2019). Victimization and perpetration of bullying/cyberbullying: Connections with emotional and behavioral problems and childhood stress. Psychosocial Intervention, 28(2): 67-73. doi: 10.5093/pi2019a3

30. González-Torres, M.L.; Vega-Campos, M.A. y Mballa Louis, V. (2017). Los avatares de las acciones gubernamentales que combaten el acoso escolar en el estado de San Luis Potosí. European Scientific Journal, ESJ, 13(29).

https://doi.org/10.19044/esj.2017.v13n29p35 
31. Inglés, C.J., Torregosa, M.S., García-Fernández, J.M., MartínezMonteagudo, M.C., Estévez, E. y Delgado, B. (2014). Conducta agresiva e inteligencia emocional en la adolescencia. Eur. J. Educ. Psychol., 7(1), 29-41. doi: 10.30552/ejep.v7i1.97

32. León, B., Gozalo, M. y Polo, M.I. (2012). Aprendizaje cooperativo y acoso entre iguales. Infancia y aprendizaje, 35(1), 23-35. https://www.tandfonline.com/doi/abs/10.1174/021037012798977494 ?journalCode=riya20

33. Menesini, E., and Salmivalli, C. (2017). Bullying in schools: the state of knowledge and effective interventions. Psychology, Health\& Medicine, 22:sup1, 240-253.

https://www.researchgate.net/publication/312869444_Bullying_in_sc hools_the_state_of_knowledge_and_effective_interventions

34. Mizuta, A., Okada, E. Nakamura, M., Yamaguchi, H. y Ojima, T. (2018). Association between the time perspective and type of involvement in bullying among adolescents: A cross-sectional study in Japan. Japan Journal of nursing science, 15(2), 156-166. https://www.researchgate.net/publication/319956850_Association_be tween_the_time_perspective_and_type_of_involvement_in_bullying _among_adolescents_A_cross-sectional_study_in_Japan

35. Modecki, K. L., Minchin, J., Harbaugh, A.G., Guerra, N.G. and Runions, K.C. (2014).Bullying prevalence across contexts: A metaanalysis measuring cyber and traditional bullying.Journal of Adolescent Health, 55(5), 602-611. doi:

10.1016/j.jadohealth.2014.06.007

36. Moore, S.E., Norman, R.E., Suetani, S., Thomas, H.J., Sly, P.D., and Scott, J.G. (2017). Consequences of bullying victimization in childhood and adolescence: A systematic review and meta-analysis. World Journal of Psychiatry, 7(1), 60-76. doi: 10.5498/wjp.v7.i1.60

37. Navarro, R., and Serna, C. (2016).Spanish youth perceptions about cyberbullying: Qualitative research into understanding cyberbullying and the role that parents play in its solution. En R. Navarro, S. Yubero and E. Larrañaga. (Eds.), Cyberbullying Across the Globe. Gender, family and mental health. (pp. 193-218). Springer International Publishing. doi: 10.1007/978-3-319-25552-1_10

38. Orue, I., and Calvete, E. (2019). Psychopathic Traits and Moral Disengagement Interact to Predict Bullying and Cyberbullying Among Adolescents. J. Interpers. Violence, 34(11), 2313-2332. doi: $10.1177 / 0886260516660302$

39. Ortega-Ruiz, R., Rey, R. D., and Casas, J. A. (2012). Knowing, building and living together on internet and social networks: The ConRed cyberbullying prevention program. International Journal of 
Computer Vision, 6(2), 302-312. https://hdl.handle.net/11441/73655 40. Perren, S., and Gutzwiller-Helfenfinger, E. (2012) Cyberbullying and traditional bullying in adolescence: Differential roles of moral disengagement, moral emotions and moral values. European Journal of Developmental Psichology, 9(2), 195-209. doi:

10.1080/17405629.2011.643168

41. Prodócimo, E., Cerezo, F. y Arense, J.J. (2014). Acoso escolar: variables sociofamiliares como factores de riesgo o de protección. Psicología Conductual, 22(2), 345-359.

https://www.behavioralpsycho.com/producto/acoso-escolarvariables-sociofamiliares-como-factores-de-riesgo-o-de-proteccion/

42. Ribeiro, N.A. and Caliman, G. (2015). The battle against cyberbullying: a discursive analysis of public policies in Brasil. European Scientific Journal, ESJ, 11(31). https://eujournal.org/index.php/esj/article/view/6626

43. Sáenz Chaparro, S.M. y Vergara Díaz, J.R. (2016). Bullying en estudiantes de Secundaria de las instituciones Educativas estatales de la UGEL 07 y nivel de preparación del Docente para abordarlo. Revista EDUCA UMCH, 8, 117-136.

https://revistas.umch.edu.pe/EducaUMCH/article/view/44

44. Triviño García, S., Iriarte Corpas, N. y González Gris, S. (2017). Perspectiva actual del bullying y la violencia escolar. En M.M. Molero. (Ed.), Salud y cuidados durante el desarrollo, Volumen I (pp. 131138). España: ASUNIVEP.

45. Van Ryzin, M.J. and Roseth, C.J. (2018).Cooperative learning in middle school: A means to improve peer relations and reduce victimization, bullying, and related outcomes. Journal of Educational Psychology, 110(8), 1192-1201. doi: 10.1037/edu0000265

46. Vilá, R., Torrado, M., and Reguant, M. (2019). Análisis de regresión lineal múltiple con SPSS: un ejemplo práctico. Revista d'Innovació $i$ Recerca en Educació, REIRE, 12(2), 1-10. doi:

10.1344/reire2019.12.222704

47. Villanueva, L., Górriz, A.B. y Adrián, J.E. (2008). El acoso escolar y el autoconcepto de agresores, defensores de la víctima y público implicado. International Journal of Developmental and Educational Psychology, 2(1), 169-175. https://www.redalyc.org/pdf/3498/349832317018.pdf

48. Wang, L., and Ngai, S. S. (2020). The effects of anonymity, invisibility, asynchrony, and moral disengagement on cyberbullying perpetration among school-aged children in China. Child. Youth Serv. Rev., 119, 105613. doi: 10.1016/j.childyouth.2020.105613 
European Scientific Journal, ESJ ISSN: 1857-7881 (Print) e - ISSN 1857-7431 February 2022 Bridging Language, Medicine, and Law

49. Yudes Gómez, C., Baridon Chauvie, D. y González Cabrera, J.M. (2018). Ciberacoso y uso problemático de Internet en Colombia, Uruguay y España: Un estudio transcultural. Comunicar, 26(56), 4958. doi: 10.3916/C56-2018-05 